\title{
IMMERSED PROJECTIVE PLANES IN LENS SPACES
}

\author{
J. SCOTT CARTER
}

(Communicated by Frederick R. Cohen)

\begin{abstract}
The minimum number of triple points of a substantial generic immersion of a projection plane in a lens space of type $(2 k, q)$ depends quadratically on $k$.
\end{abstract}

\section{INTRODUCTION}

(1.1) History. In [1], Bredon and Wood give a lower bound for the genus of a nonorientable surface embedded in a lens space of type $(2 k, q)$. This bound is bounded below by a linear function of $k$. In [3], the present author and Ki Hyoung Ko gave an example of an immersed projective plane in $L(4,1)$ that represents a 2-dimensional modulo 2 homology class. This example was important to the proof of triple point formulas.

During a seminar in which the author presented the results of [3], John Wood asked if the minimal number of triple points of an immersed projective plane in a $(2 k, q)$-lens space would grow as a function of $k$. He and the author constructed some examples that suggested this function is at least quadratic. Indeed this is the case: Theorem 2.6 and Theorem 3.1.

The key steps of section 2 were indicated to the author in a brief conversation with Peter Scott.

(1.2) Organization. Section 2 contains results on lifting maps of projective planes into lens spaces up to maps into real projective 3-space. Information on the intersection sets is contained in the intersection of various lifts. Theorem 2.6 gives a lower bound on the number of triple points.

Section 3 contains results on when the lower bound can be achieved. Many examples are given here.

The chief result is the following:

(1.3) Theorem. A general position immersion $f: P^{2} \rightarrow L(2 k, q)$ such that $f_{*} \neq 0$ on $H_{2}(-; \mathbf{Z} / 2)$ has at least $\left\lceil\left(\begin{array}{l}k \\ 3\end{array}\right)(1 / k)\right\rceil$ triple points where $\lceil x\rceil$ is Knuth's ceiling function defined by $\lceil x\rceil=-$ greatest integer $(-x)$.

Received by the editors March 28, 1988 and, in revised forms, September 8, 1988 and October 21, 1988.

1980 Mathematics Subject Classification (1985 Revision). Primary 57R42; Secondary 57M35.

(C) 1989 American Mathematical Society $0002-9939 / 89 \$ 1.00+\$ .25$ per page 


\section{LifTING SUBSTANTIAL MAPS OF THE PROJECTIVE PLANE}

A map $f: X^{2} \rightarrow Y^{3}$ from a closed surface $X$ to a 3-manifold $Y$ will be said to be substantial if $f_{*}: H_{2}(X ; \mathbf{Z} / 2) \rightarrow H_{2}(Y ; \mathbf{Z} / 2)$ is a non-trivial homomorphism. Equivalently, the image of the fundamental homology class is non-trivial.

(2.1) Lemma. If $f: P^{2} \rightarrow L(2 k, q)$ is a substantial map, then the induced homomorphism, $\pi_{1}(f)$, on the fundamental group is a monomorphism.

Proof. If $\pi_{1}(f)$ is trivial, then the map $f$ lifts to a map of $P^{2}$ into $S^{3}$ which is the universal cover of $L(2 k, q)$. This contradicts substantiality.

(2.2) Lemma. Suppose that $f: P^{2} \rightarrow L(2 k, q)$ is substantial. Then there is a lift $\tilde{f}: P^{2} \rightarrow \mathbf{R} P^{3}$ to the $k$-fold covering of $L(2 k, q)$. Any such lift is substantial. Proof. Let $p: \mathbf{R} P^{3} \rightarrow L(2 k, q)$ denote the covering projection. Then the images of $\pi_{1}(f)$ and $\pi_{1}(p)$ coincide. Thus a lift exists by the results in [4]. Since $p \tilde{f}=f$ and $f$ is substantial, any lift, $\tilde{f}$ of $f$, is also substantial.

Consider the pull back,

$$
f^{*}\left(\mathbf{R} P^{3}\right)=\left\{(x, y) \in P^{2} \times \mathbf{R} P^{3}: f(x)=p(y)\right\},
$$

of the principal $\mathbf{Z} / k$ fibration $p: \mathbf{R} P^{3} \rightarrow L(2 k, q)$.

(2.3) Lemma. Suppose that $f: P^{2} \rightarrow L(2 k, q)$ is substantial. The principal $\mathbf{Z} / k$ fibration $f^{*}\left(\mathbf{R} P^{3}\right) \rightarrow P^{2}$ is trivial.

Proof. By Lemma 2.1 the composition

$$
P^{2} \stackrel{f}{\rightarrow} L(2 k, q) \stackrel{B p}{\rightarrow} K(\mathbf{Z} / k, 1)
$$

is null homotopic where $B p$ is the classifying map for $\left(p, \mathbf{R} P^{3}\right)$ and $K(\mathbf{Z} / k, 1)$ is an Eilenberg-MacLane space.

(2.4) Corollary. The group $\mathbf{Z} / k$ acts on $f^{*}\left(\mathbf{R} P^{3}\right)$ by cyclically permuting the $k$ copies of $P^{2}$. Each such $P^{2}$ yields a lift of $f$.

Let $\tilde{f}: f^{*}\left(\mathbf{R} P^{3}\right) \rightarrow \mathbf{R} P^{3}$ be defined by $\tilde{f}(x, y)=y$. Let the components of $f^{*}\left(\mathbf{R} P^{3}\right)$ be denoted by $P_{1}^{2}, P_{2}^{2}, \ldots, P_{k}^{2}$, and let $\tilde{f}_{j}=\tilde{f} \mid P_{j}^{2}$ for $j=1,2, \ldots, k$. Thus $p \tilde{f}_{j}=f$ for all $j=1,2, \ldots, k$.

For each set of three distinct integers $l, m, n$ such that $1 \leq l, m, n \leq k$ the cohomology class

$$
\left[\tilde{f}_{l}\left(P^{2}\right)\right]^{*} \cup\left[\tilde{f}_{m}\left(P^{2}\right)\right]^{*} \cup\left[\tilde{f}_{n}\left(P^{2}\right)\right]^{*}
$$

is non-zero in $H^{3}\left(\mathbf{R} P^{3} ; \mathbf{Z} / 2\right)$. Here $[x]^{*}$ denotes the Poincare dual of the class represented by $x$. This 3-dimensional cohomology class is dual to the 0-dimensional class determined by the transverse intersection

$$
Z_{l, m, n}=\tilde{f}_{l}\left(P^{2}\right) \cap \tilde{f}_{m}\left(P^{2}\right) \cap \tilde{f}_{n}\left(P^{2}\right) .
$$


(2.5) Lemma. If $l, m$, and $n$ are distinct integers between 1 and $k$ inclusive, and if $f: P^{2} \rightarrow L(2 k, q)$ is a generic substantial map, then $Z_{l, m, n} \neq \varnothing$ and $Z_{l, m, n}$ contains an odd number of points. If $x \in Z_{l, m, n}$ then $p(x)$ is a triple point of $f$.

Proof. It remains to be shown that $p(x)$ is a triple point of $f$. Since $x \in$ $Z_{l, m, n}$, then $x=\tilde{f}_{l}\left(a_{1}\right)=\tilde{f}_{m}\left(a_{2}\right)=\tilde{f}_{n}\left(a_{3}\right)$ for $a_{1}, a_{2}$, and $a_{3}$ distinct; this follows by the uniqueness of lifts theorem [4]. Then $p(x)=f\left(a_{1}\right)=f\left(a_{2}\right)=$ $f\left(a_{3}\right)$ for distinct points $a_{1}, a_{2}$, and $a_{3}$. Thus $p(x)$ is a triple point.

The group $\mathrm{Z} / k$ cyclically permutes the lifts $\tilde{f}_{1}, \ldots, \tilde{f}_{k}$ of the substantial map $f$. Thus $\mathbf{Z} / k$ acts on the intersection sets $Z_{l, m, n}$. This latter action is given by

$$
Z_{l, m, n}+a:=Z_{l+a, m+a, n+a}
$$

for $a \in \mathbf{Z} / k$ and $l, m, n \in \mathbf{Z} / k$ distinct. (The arithmetic on the subscripts is modulo $k$.) Let $B(k, 3)$ denote the number of orbits under this action.

(2.6) Theorem. Any generic substantial map $f: P^{2} \rightarrow L(2 k, q)$ has at least $B(k, 3)$ triple points.

Proof. Each three-fold intersection set $Z_{l, m, n}$ contains at least one point. The number of orbit sets $B(k, 3)$ is a lower bound on the number of triple points.

An exact value for $B(k, 3)$ is computed in the following section.

(2.7) Combinatorics. Let $\left\{\left(\begin{array}{l}k \\ r\end{array}\right)\right\}$ denote $\left\{\left\{i_{1}, \ldots, i_{r}\right\} \subset\{1,2, \ldots, k\}\right.$ : $i_{1}, \ldots, i_{r}$ are distinct $\}$. Thus $\left\{\left(\begin{array}{l}k \\ r\end{array}\right)\right\}$ denotes the set of $r$-fold subsets of $\{1, \ldots, k\}$. An action of the cyclic group $\mathbf{Z} / k$ will be defined on $\left\{\left(\begin{array}{l}k \\ r\end{array}\right)\right\}$. The easiest way to define this action is to assume the constituents in $\{1,2, \ldots, k\}$ are integers modulo $k$. Then for any $a \in \mathbf{Z} / k$, define

$$
\left\{i_{1}, \ldots, i_{r}\right\}+a:=\left\{i_{1}+a, \ldots, i_{r}+a\right\}
$$

where the addition on the right is the operation of the cyclic group $Z / k$. Let $\left\{\left(\begin{array}{l}k \\ r\end{array}\right)\right\} / Z / k$ denote the set of orbits under this action; let $B(k, r)$ denote the number of orbits.

An argument that involves counting the asymmetrical elements in $\left\{\left(\begin{array}{l}k / d \\ r / d\end{array}\right)\right\}$ where $d$ divides $k$ and $r$ yields:

$$
B(k, r)=\sum_{d \mid \text { g.c.d. }(k, r)} \frac{d}{k} \sum_{\delta \mid \text { g.c.d. }\left(\frac{k}{d}, \frac{r}{d}\right)} \mu(\delta)\left(\begin{array}{l}
k / d \delta \\
r / d \delta
\end{array}\right) .
$$

Here $\mu(x)$ is the Möbius function. In case $r=3$, then this formula simplifies to $B(k, 3)=\left\lceil\left(\begin{array}{l}k \\ 3\end{array}\right)(1 / k)\right\rceil$. One may also see this by establishing a 1-1 correspondence between $\left\{\left(\begin{array}{l}k \\ r\end{array}\right)\right\} / \mathbf{Z} / k$ and $\left\{\left(v_{1}, \ldots, v_{r}\right) \in \mathbf{N}^{r}: \sum v_{i}=k\right\} / \mathbf{Z} / r$. Theorem 2.6 can be restated as Theorem 1.3.

Using the techniques of section 2, similar lower bounds can be obtained on the number of zero dimensional multiple points of immersed projective $n$ spaces. These are discussed in the paper [2]. 


\section{EXAMPLES}

For each pair of integers $k$ and $q$ with $q$ relatively prime to $2 k$ an immersion $\tilde{f}: \coprod_{j=1}^{k} P_{j}^{2} \rightarrow \mathbf{R} P^{3}$ is constructed that projects to an immersion $f: P^{2} \rightarrow L(2 k, q)$. At first this immersion $\tilde{f}$ is not in general position. There is a general position approximation to $\tilde{f}$ that has $\left(\begin{array}{l}k \\ 3\end{array}\right)$-triple points. It can be shown for $k=4$ and 5 that this immersion is not equivariant under the action $\mathbf{Z} / k$ that yields $L(2 k, 1)$. If 3 divides $k$, then the immersion with $\left(\begin{array}{l}k \\ 3\end{array}\right)$ triple points cannot be equivariant under any $\mathbf{Z} / k$ action.

The results of the section are summarized in the following:

(3.1) Theorem. Let $f: P^{2} \rightarrow L(2 k, q)$ denote a substantial immersion in general position. Suppose that $f$ has the minimal number of triple points among all such immersions. Let $T(f)$ denote the number of triple points. Then

$$
\left\lceil\frac{1}{k}\left(\begin{array}{l}
k \\
3
\end{array}\right)\right\rceil \leq T(f) \leq q\left(\begin{array}{c}
k-q \\
2
\end{array}\right)+\left(\begin{array}{l}
q \\
3
\end{array}\right) .
$$

(3.2) The action of $\mathbf{Z} / k$. The easiest way to visualize the action of $\mathbf{Z} / k$ on $\mathbf{R} P^{3}$ that yields $L(2 k, q)$ as quotient is to consider $\mathbf{R} P^{3}$ as a lens space of type $(2 k, k q)$. That is, to a solid torus $k$ solid 2-handles are attached each along a $(2, q)$ torus curve on the boundary. The boundary of the result is the union of $k$ disjoint 2-spheres. Solid 3-handles are attached along these. Then the group $\mathrm{Z} / k$ acts by cyclically permuting the 2 and 3 handles among themselves. The solid torus is rotated longitudinally through an angle of $2 \pi / k$. Henceforth, the notations $L(2 k, k q)$ and $\mathbf{R} P^{3}$ will both be used to denote the real projective 3-space.

(3.3) The caduceus. In $L(2 k, k q)$ cut the solid torus piece along a meridonal disk. Then the attaching spheres for the 2-handles form caduceus-like or barber pole-like curves winding from top to bottom. If each attaching region is labeled with an integer in $\{1,2, \ldots, k\}$, then $2 k$ curves result on $S^{1} \times I$ that are paired antipodally. In any meridonal disk join pairs of antipodal points by a diameter of the disk. Each arc (or snake) of the caduceus winds $q$ half turns meridonally as the arc moves from bottom to top. Thus the union of a pair of antipodal arcs and the diameter joining them forms an embedded disk in $D^{2} \times I$. On any one of these disks the top and bottom diameters are identified in an orientation reversing pattern. An embedded Möbius band results.

(3.4) Theorem. An immersion $\tilde{f}: \bigsqcup_{j=1}^{k} P_{j}^{2} \rightarrow L(2 k, k q)$ exists that is equivariant under the action of $\mathbf{Z} / k$ which yields $L(2 k, q)$ as quotient. Each component of the domain of $\tilde{f}$ is embedded.

Proof. To the embedded Möbius bands above attach the cores of the solid 2handles.

(3.5) Putting $\tilde{f}$ in general position. The following construction is essentially due to John Wood with minor modifications by the author. 
The immersion $\tilde{f}$ has an arc of $k$-tuple points. Unless $k=2$, this immersion is not in general position. In most meridonal disks of the solid torus the diameters are to be jostled into general position. Those disks in which the diameters are not generic will have the property that at most three arcs are coincident; the "nongeneric" disks form a finite set. To see the general position version of $\tilde{f}$, consider the intersection pattern of curves on $\partial\left(D^{2} \times I\right)$ that is given by $\tilde{f}$. This is depicted in Figure 3.5.1 for $k=2, q=1$ and in Figure 3.5.2 for $k=4, q=3$.

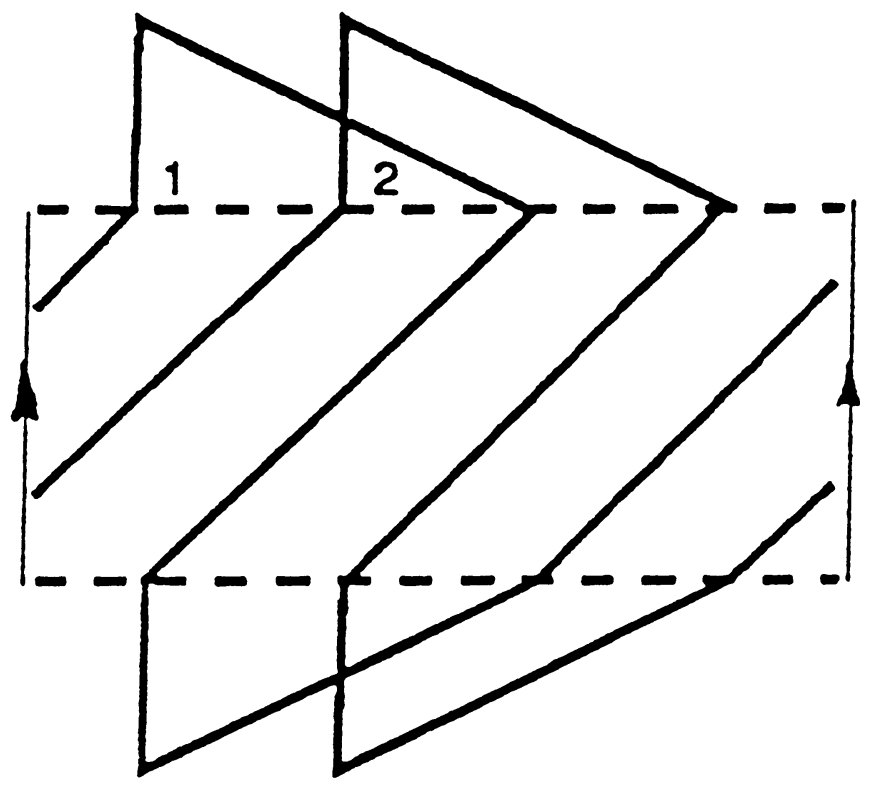

FIGURE 3.5.1. $P^{2} \amalg P^{2} \rightarrow L(4,2)$

In these figures, the tropical region, $S^{1} \times I$, of the boundary has been cut open along an arc $\{z\} \times I$. Portions of the polar regions are deleted. These figures are analogous to the standard planar maps of the earth.

In the polar regions the diameters have been put into general position in the following way. Each diameter is labeled with an integer in the set $\{1,2, \ldots, k\}$. These labels are given in order at one end of the arcs. This determines an orientation for the arc. Then for each labeled arc, the remaining arcs intersect in order. Thus on the 1st diameter the remaining diameters intersect in the order $\{2,3, \ldots, k\}$, on the second the intersection sequence is $\{1,3,4, \ldots, k\}$ and so forth.

(3.6) Lemma. Let $\tilde{f}: \coprod_{j=1}^{k} P_{j}^{2} \rightarrow L(2 k, k q)$ denote the general position immersion constructed in section 3.5. Then $\tilde{f}$ has $\left(\begin{array}{l}k \\ 3\end{array}\right)$ triple points. 


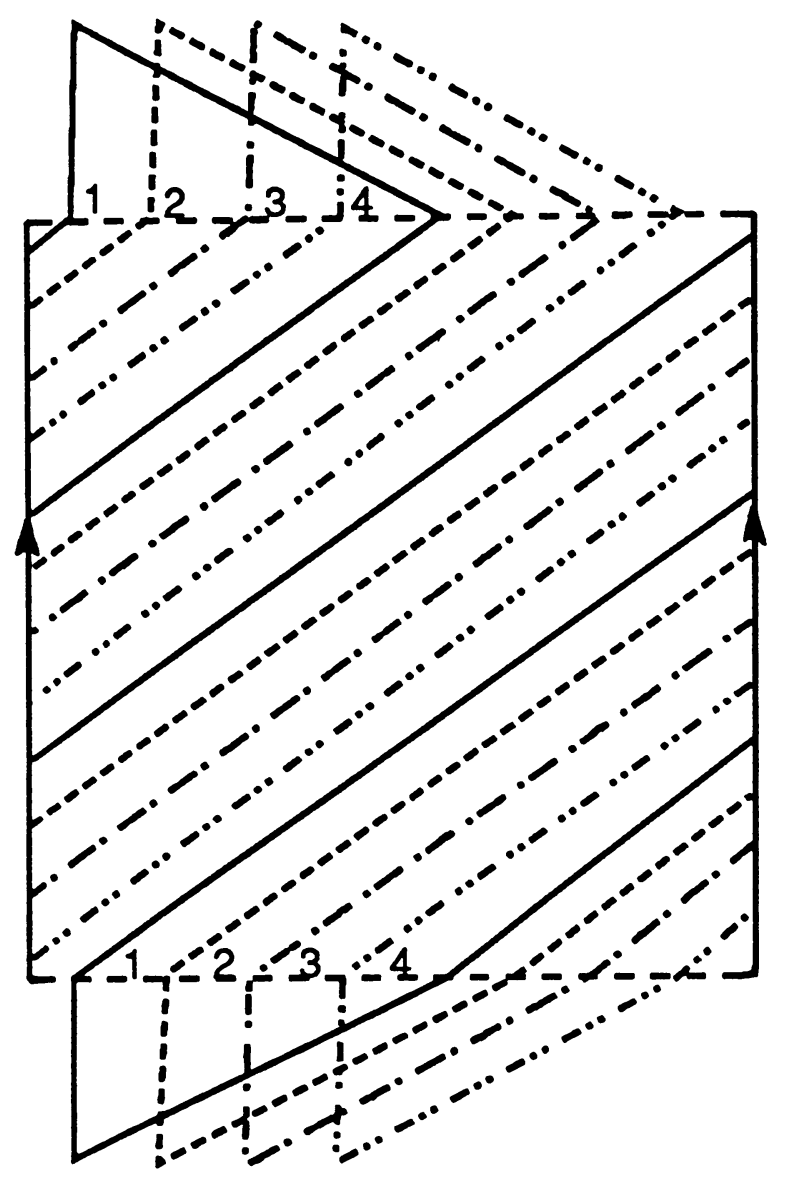

FIGURE 3.5.2.

Proof. Each simple closed curve on $\partial\left(D^{2} \times I\right)$ bounds two regions. Each contains $\left(\begin{array}{c}k-1 \\ 2\end{array}\right)$ double points. This number is independent of $q$. A disk bounded by such a curve is obtained as a vertical collar of the curve together with a flat mesa. The height of the mesa is $n \varepsilon$ for the $n$th curve. The first such mesa, then, intersects the double points in $\left(\begin{array}{c}k-1 \\ 2\end{array}\right)$ points. By induction, the total number of triple points is

$$
\left(\begin{array}{c}
k-1 \\
2
\end{array}\right)+\left(\begin{array}{c}
k-2 \\
2
\end{array}\right)+\cdots+\left(\begin{array}{l}
2 \\
2
\end{array}\right)=\left(\begin{array}{l}
k \\
3
\end{array}\right)
$$

This completes the proof. 
Unfortunately, this immersion is not always equivariant. Moreover, the only uniform method for finding an equivariant, general position version of this is to construct examples in the quotient space.

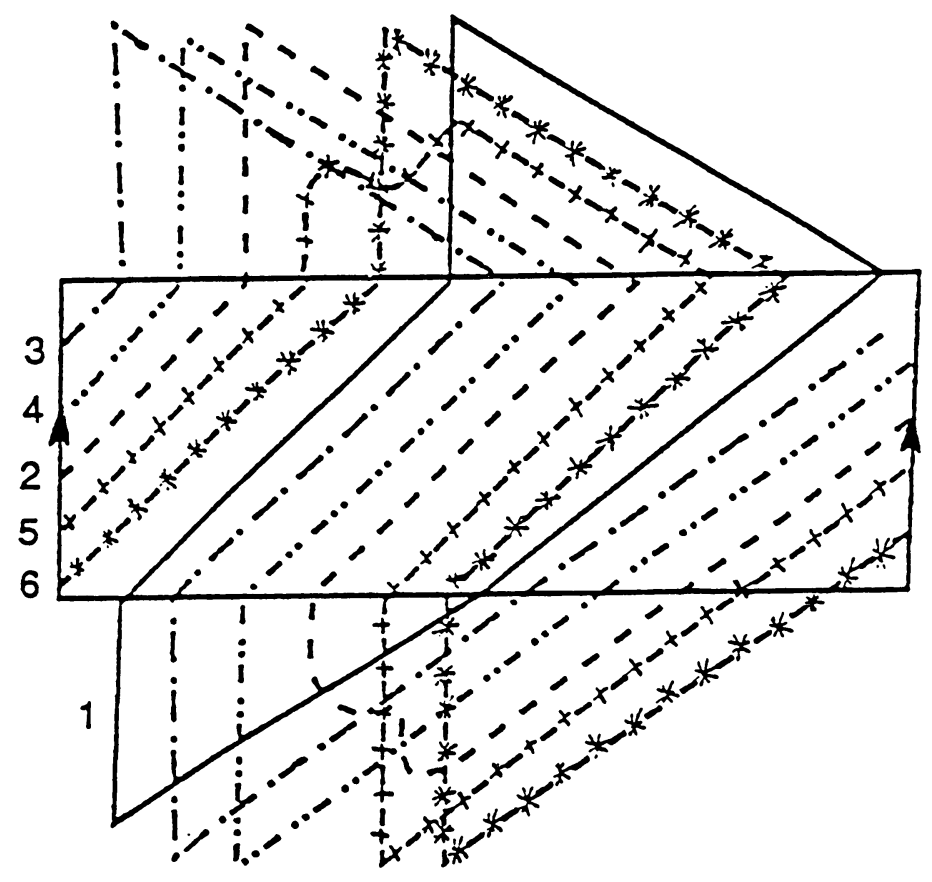

FIGURE 3.7.1. $P^{2} \rightarrow L(12,5)$ with 6 triple points

(3.7) The case 3 divides $k$. If $k=3 l$ for some integer $l$, then there is an exceptional orbit of the action of $\mathbf{Z} / k$ on $\left\{\left(\begin{array}{l}k \\ 3\end{array}\right)\right\}$. A correspondence between the set $\left\{\left(\begin{array}{l}k \\ 3\end{array}\right)\right\}$ and the intersection sets

$$
Z_{l, m, n}=\left\{\tilde{f}\left(P_{l}^{2}\right) \cap \tilde{f}\left(P_{m}^{2}\right) \cap \tilde{f}\left(P_{n}^{2}\right): l \neq m \neq n \neq l\right\}
$$

has been given. An intersection set with non-zero stabilizer has $k / 3=l$ sets in its orbit. Thus the immersion $\tilde{f}$ constructed in Lemma 3.6 cannot be equivariant, for $\mathbf{Z} / k$ should act freely on the collection of triple points.

In Figure 3.7.1 an example, due to the referee, is illustrated. This yields an immersed $P^{2} \rightarrow L(12,5)$ with 6 triple points. The value 6 is greater than the lower bound given by (1.4) and is much better than the upper bound in Theorem 3.8 .

(3.8) Theorem. There is an immersion $f: P^{2} \rightarrow L(2 k, q)$ with $q\left(\begin{array}{c}k-q \\ 2\end{array}\right)+\left(\begin{array}{l}q \\ 3\end{array}\right)$ triple points. 
Proof. The Figures 3.9.3 and 3.9.4 can be generalized as follows. The snakes of the caduceus will have slope $1 / q$. The slopes of the non-vertical lines at the top of the figures are $-1 / k$; the slopes of the non-vertical lines at the bottom are $1 / k$. The curves bound mesas of increasing height starting with the curve that contains the bottom-most point on the far left. To complete the proof count the number of double points that are enclosed by each curve after the preceding curves have been removed. The sum of the double points enclosed is as indicated. The proofs of Theorem 3.1 and 3.8 are complete.

Improvements can be made as in Figures 3.9.1, 3.9.2, and 3.7.1. So far the techniques to make these improvements have been ad hoc.

(3.9) Final examples. Figures 3.9.1 and 3.9.2 yield immersions in $L(8,3)$ and $L(10,3)$ with 1 and 2 triple points, respectively. Figures 3.9 .3 and 3.9.4 yield immersions in $L(8,1)$ and $L(10,1)$ with 3 and 6 triple points, respectively. A tedious calculation shows these latter two immersions achieve the minima.

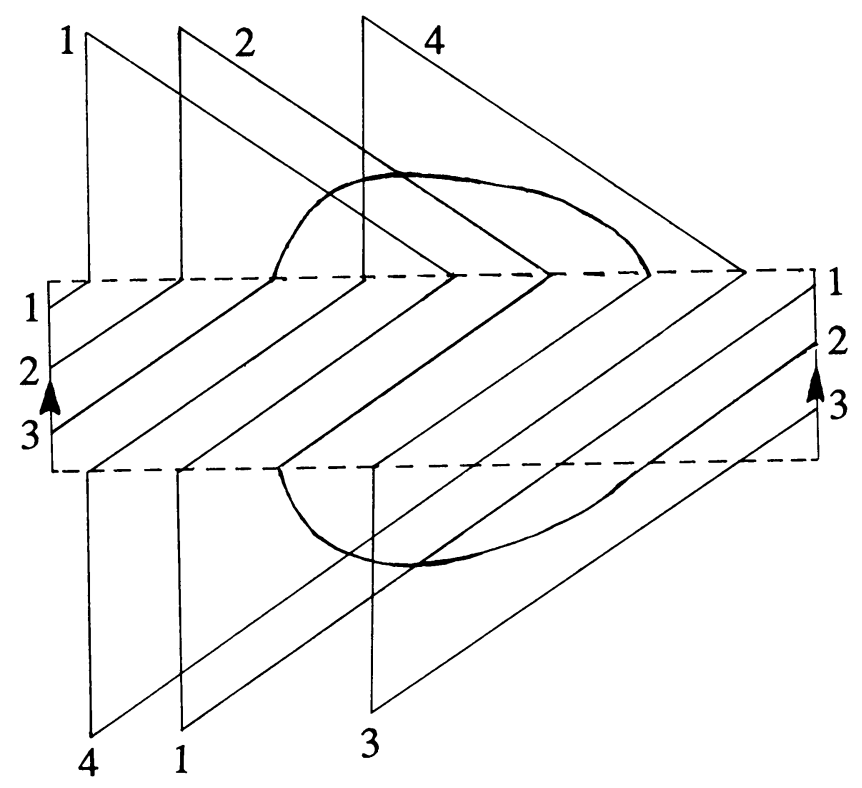

FIGURE 3.9.1. $P^{2} \rightarrow L(8,3)$ with one triple point 


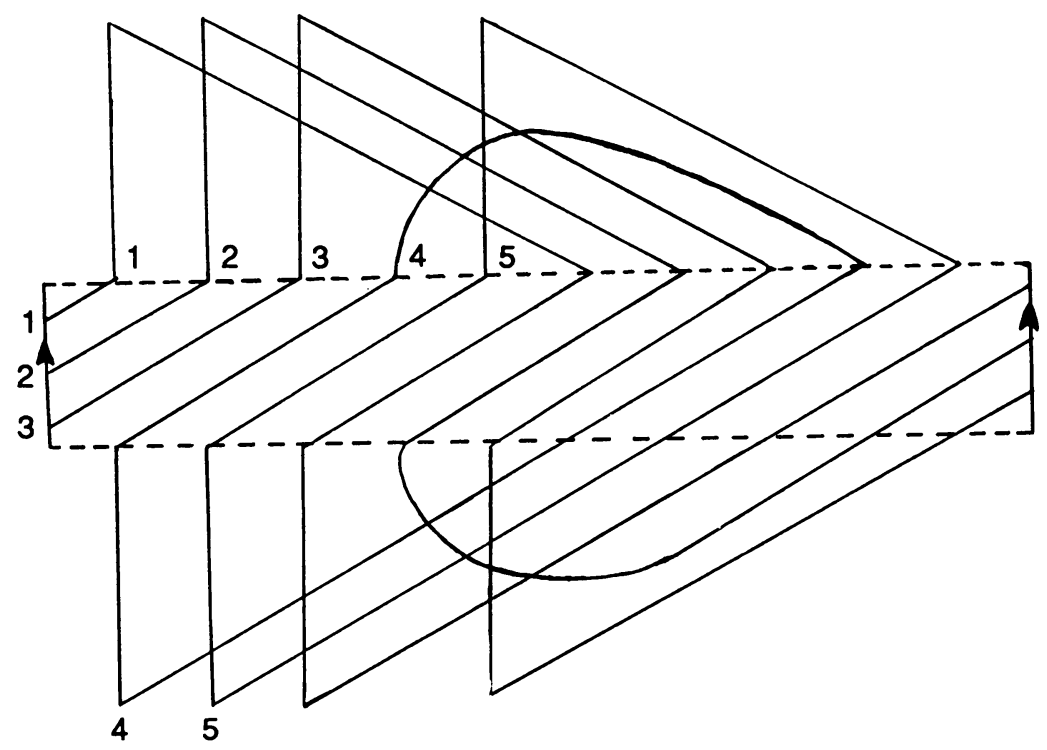

FIGURE 3.9.2. $P^{2} \rightarrow L(10,3)$ with two triple points

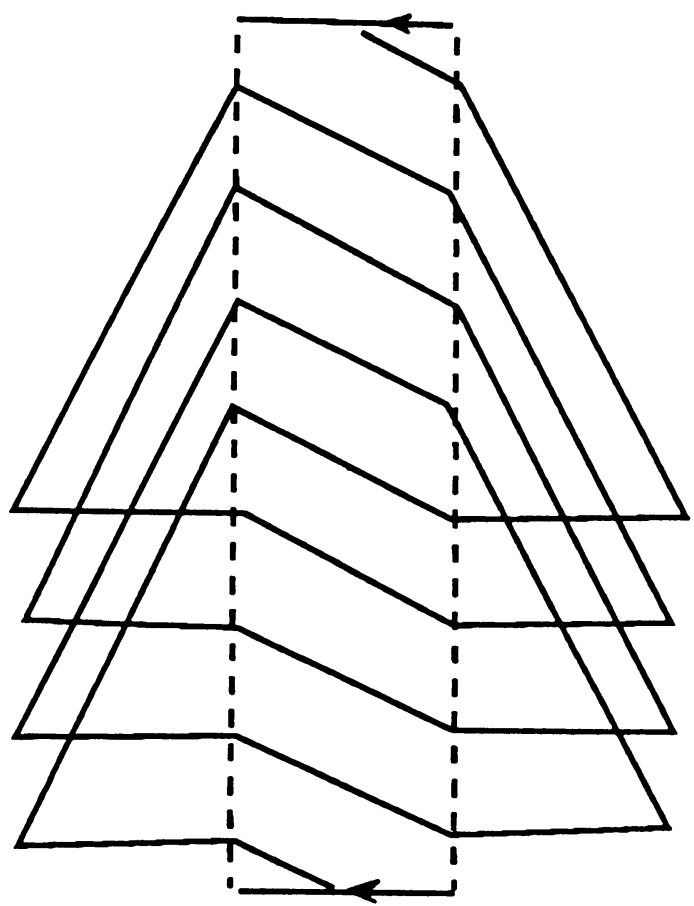

FIGURE 3.9.3. $P^{2} \rightarrow L(8,1)$ with three triple points 


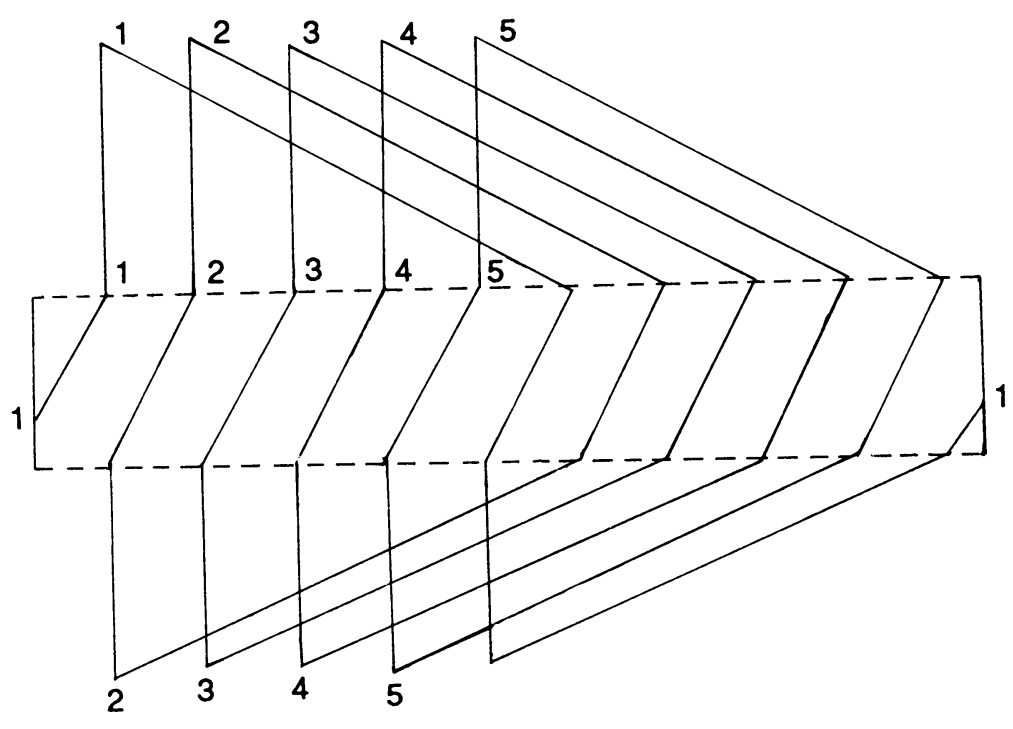

FIGURE 3.9.4. $P^{2} \rightarrow L(10,1)$ with six triple points

\section{ACKNOWLEDGMENT}

The author gratefully acknowledges the following three sources. First, John Wood pointed out the problem to me and was a great deal of help in constructing the examples of section 3. Second, Peter Scott pointed out the methods of section 2. Finally, the author was supported by a summer grant from Lake Forest College while some of the research in this project was being conducted.

\section{REFERENCES}

1. G. Bredon, and J. Wood, Non-orientable surfaces in orientable 3-manifolds, Inventiones Math. 7 (1969), 83-110.

2. J. Scott Carter, Immersed codimension one projective spaces in spherical space forms, To appear in the Proceedings of the AMS.

3. Ki Hyoung Ko and J. Scott Carter, Triple points of immersed surfaces in three-dimensional manifolds, (to appear).

4. Wm. S. Massey, “Algebraic topology : an introduction”, Springer, GTM56, 1967.

Department of Mathematics, The University of Texas at Austin, Austin, Texas 78712

Current address: Department of Mathematics, Wayne State University, Detroit, Michigan 48202 